
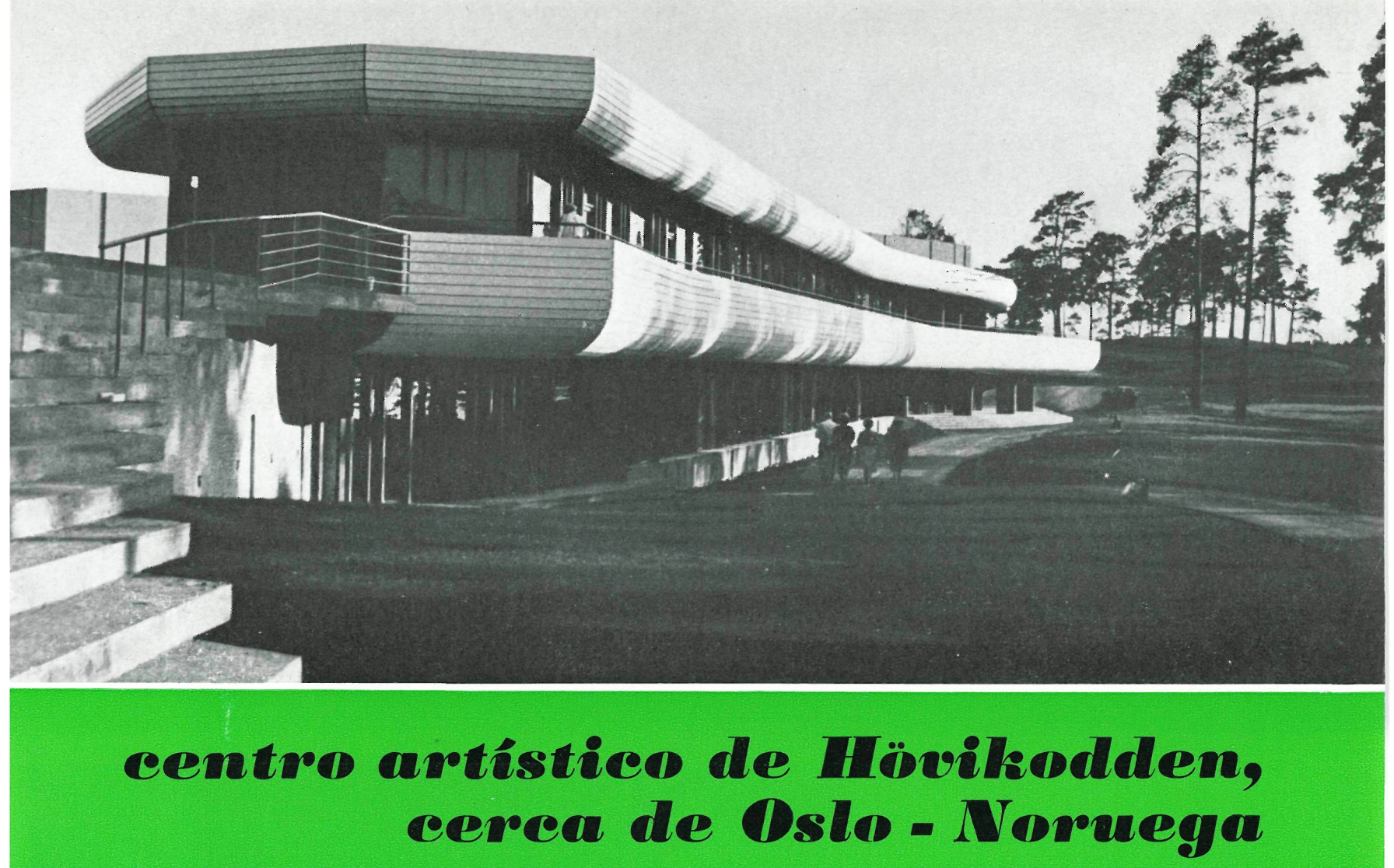

EIKVAR y ENGEBRETSEN, arquitectos

sipnosis

$181-2$

A unos $12 \mathrm{~km}$ de Oslo ha sido erigido este gran Centro artístico, destinado no solamente a la exhibición de obras plásticas, sino tam. bién a la investigación de todas las ramas del arte contemporáneo. Entre sus instalaciones figuran: Museo, Teatro, Sala de conciertos y conferencias, Biblioteca, Saia de estudios, asi como alojamientos para los estudiantes becarios y empleados. - Para componer el conjunto los arquitectos jugaron con una serie de bloques masivos, en los que contrastan la sensacion de "pesadezn que sugieren los muros, y la de uligezais de las cubiertas; logrando una composish las diversas artes se hace tangible $\mathrm{y}$ un lugar ampliamente abierto a intercambios de contactos entre tendencias muy diversas.

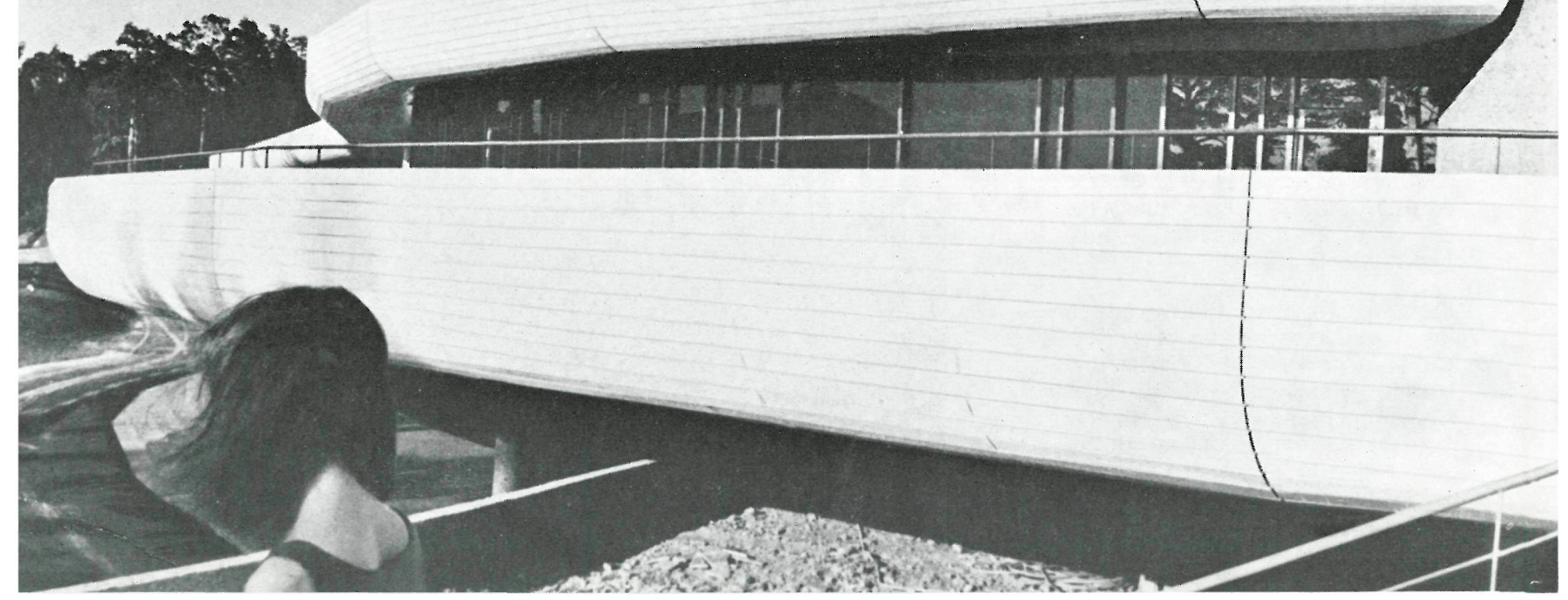




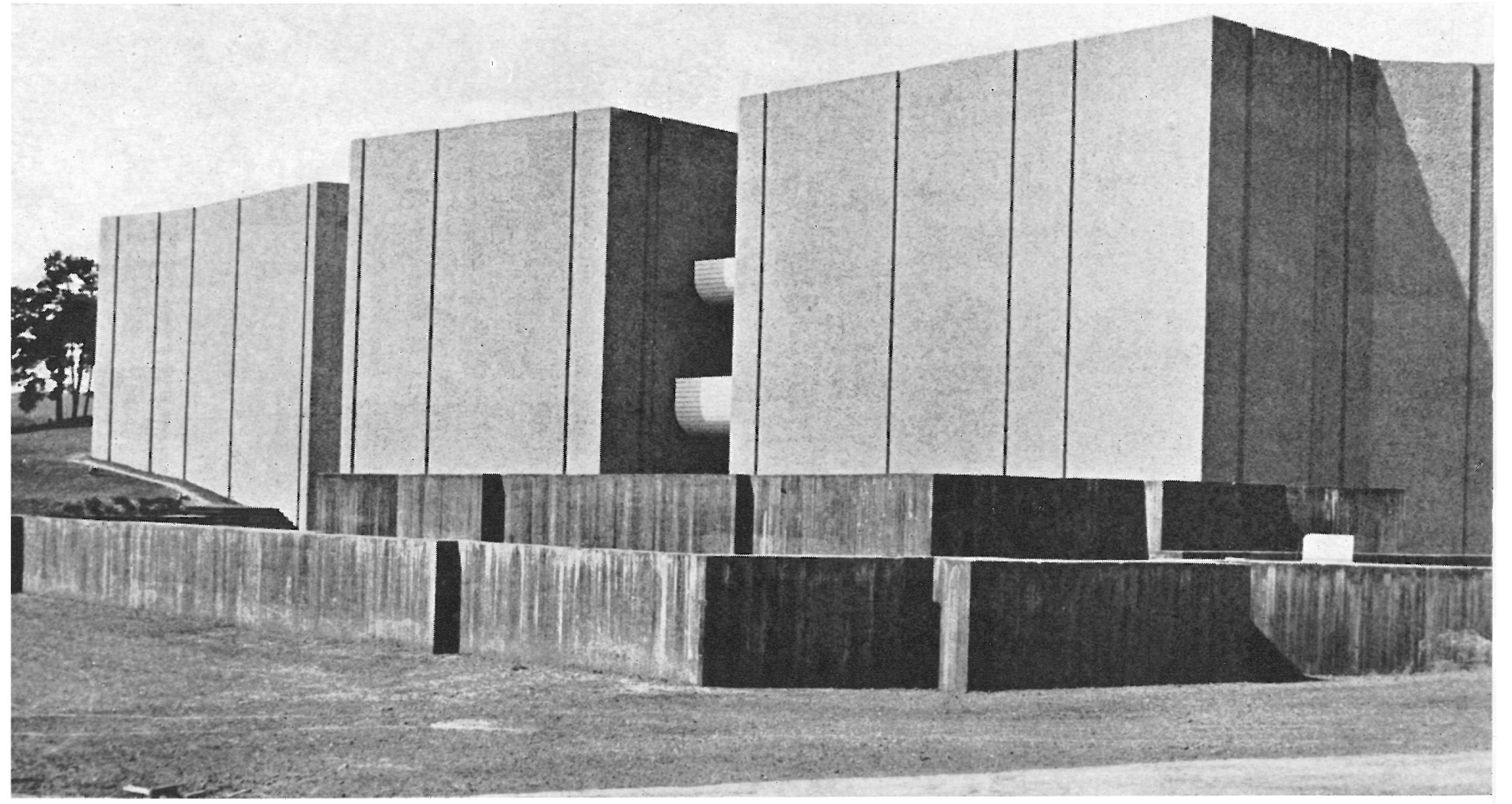

La Fundación creada por el matrimonio Sonja Henie y Niels Onstad ha promovido la construcción de un Museo, con el fin de que no solamente albergara una parte muy importante de su colección de obras plásticas, sino que, al mismo tiempo, constituyera un importante Centro de actividades en todas las ramas artísticas: Teatro, Música, Danza, Cine, Literatura, Arquitectura y Artes aplicadas.

El Ayuntamiento de Bærum, comprendiendo lo que significaría para la ciudad un Centro cultural de tal envergadura, no sólo cedió el terreno idóneo, sino que se encargó de urbanizar los alrededores en uno de los sitios más agradables disponibles. Se llama Hövikodden, paraje idílico y lugar de retiro muy apreciado: una posesión de unas $14 \mathrm{Ha}$, situada en una punta de tierra que penetra en el fiord de Oslo, a unos $12 \mathrm{~km}$ de la capital.

Para su proyecto influyeron en los arquitectos, entre otros factores: su anhelo de lograr una composición arquitectónica libre y dinámica, pero sin perder el sentido de unidad del conjunto;
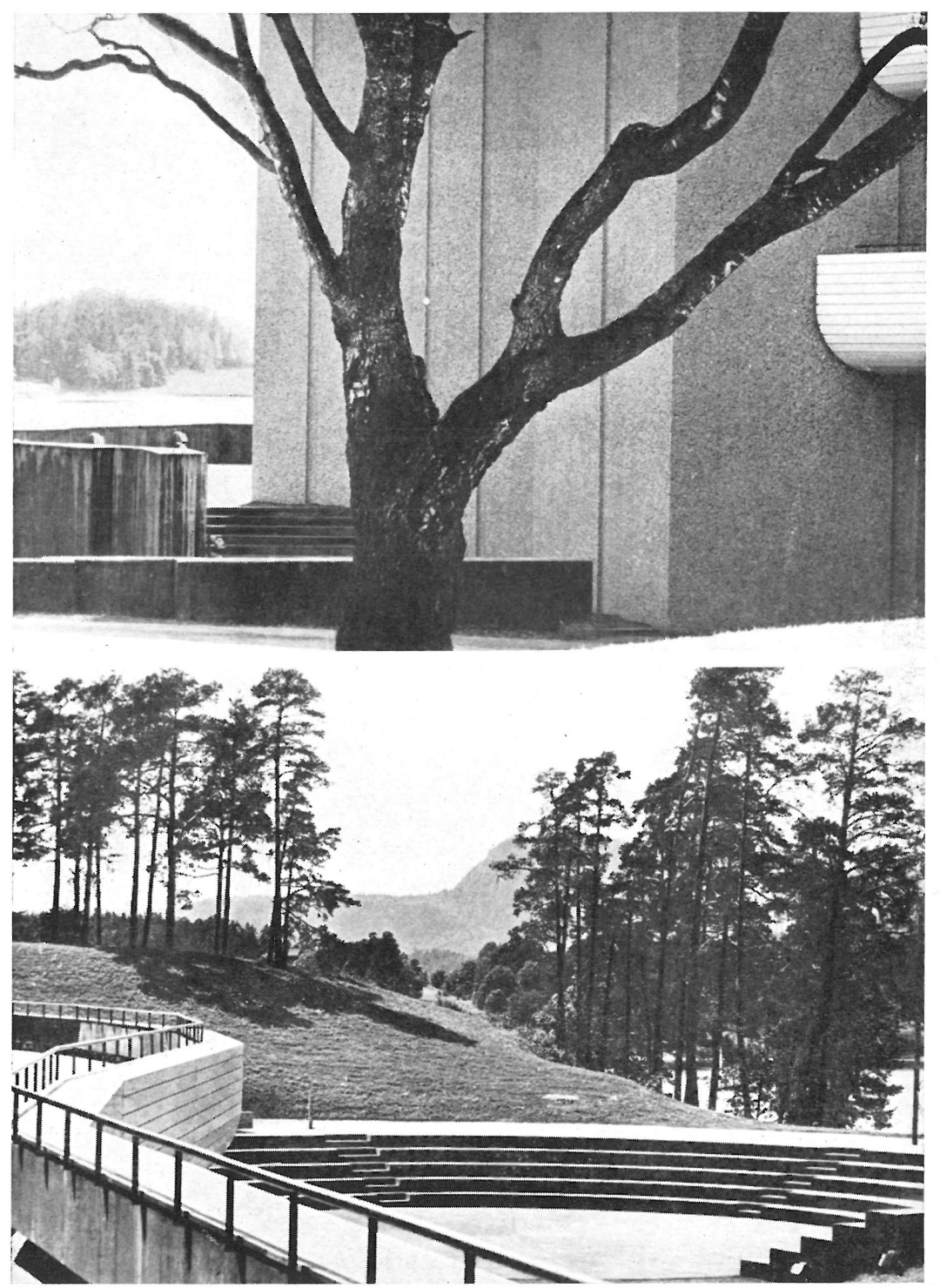


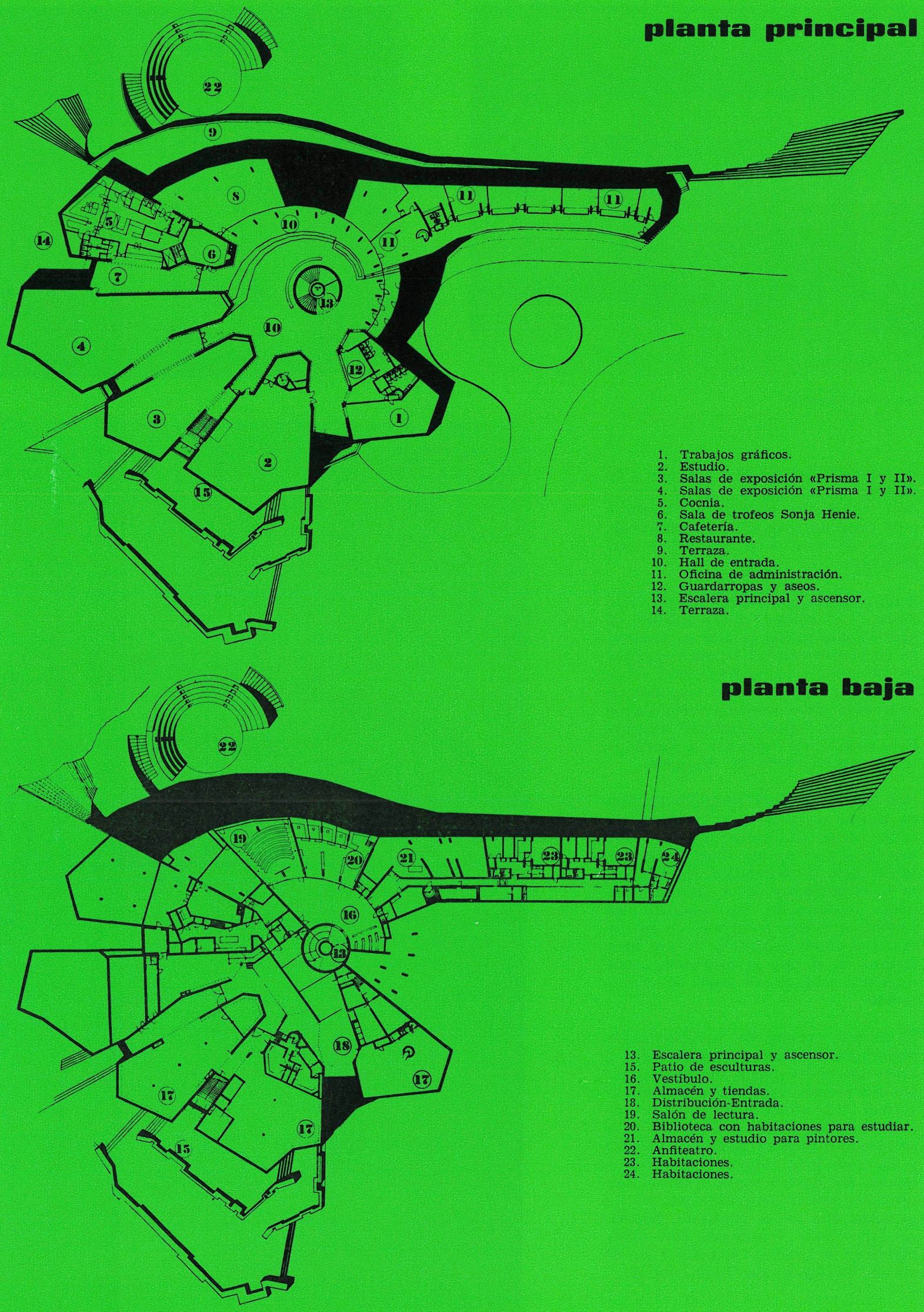



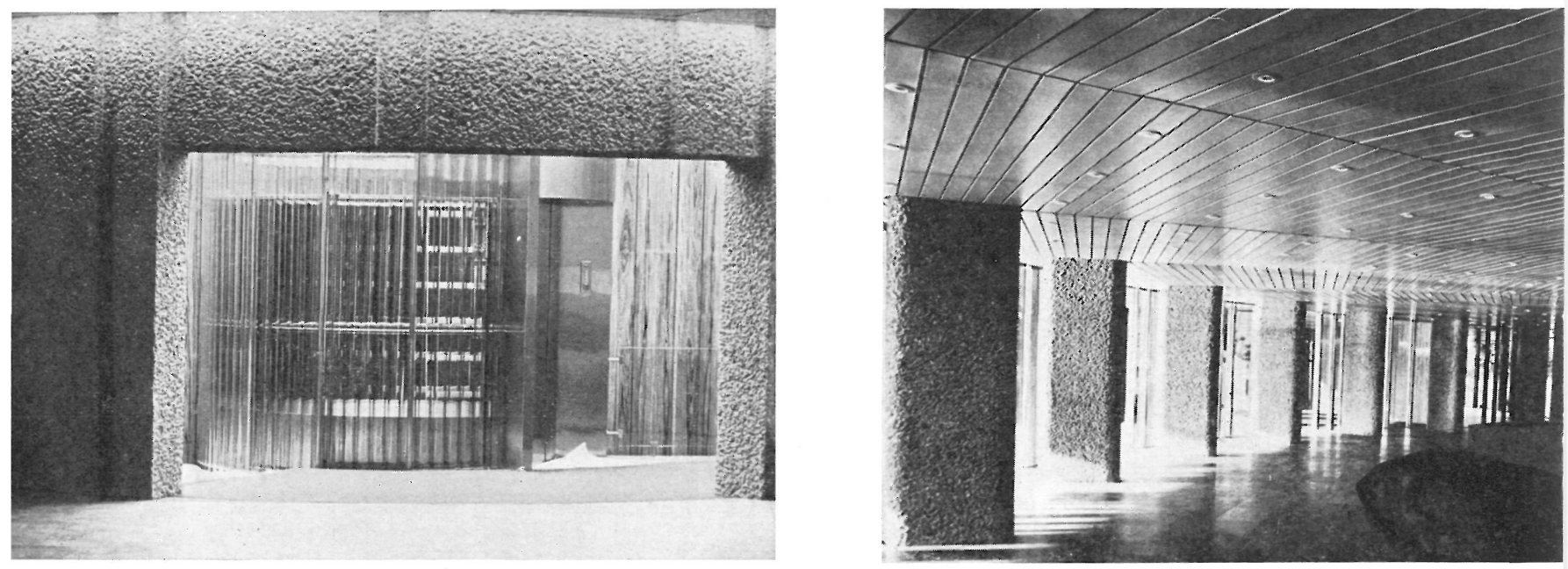

Fotos: EIKVAR Y ENGEBRETSEN

la fisonomía del paisaje; y el deseo de plasmar los propósitos de la Fundación: crear una Institución abierta a toda clase de contactos, y cambios de puntos de vista entre tendencias muy diversas.

A tal respecto los autores del proyecto dicen: «... influyó decisivamente en nuestra inspiración la idea de facilitar un encuentro entre el artista y el público, no como una confrontación sobre un terreno neutral, con el resultado de su trabajo, sino como una participación colectiva en el procedimiento creativo. Esta inspiración engendró, naturalmente, un nuevo modo de expresión.

Nos hizo falta, pues, imaginar un foro en el que el parentesco entre las diversas Artes se hiciese tangible. Tuvimos la visión de una Institución que se dirigiese y trascendiese directamente en el público y no se limitase a exponer pasivamente sus tesoros; esta visión nos llevó a componer un cuadro material como expresión de esa idea cuadro en el que la expresión arquitectónica debía de ser digna de su contenido.

El aspecto externo de la construcción seguramente inducirá al espectador a creer que no es sino la materialización de una visión; ello no es totalmente verdad. Una "forma» en arquitectura, pensamos, no tiene ninguna razón de ser, si no está estrechamente asociada y adaptada a la función; y este pensamiento nos llevó a tratar la forma definitiva de nuestro edificio como un organismo que en el conjunto, además de ligar unos volúmenes con otros, ofreciese un marco adecuado a las diversas funciones que iba a cobijar.

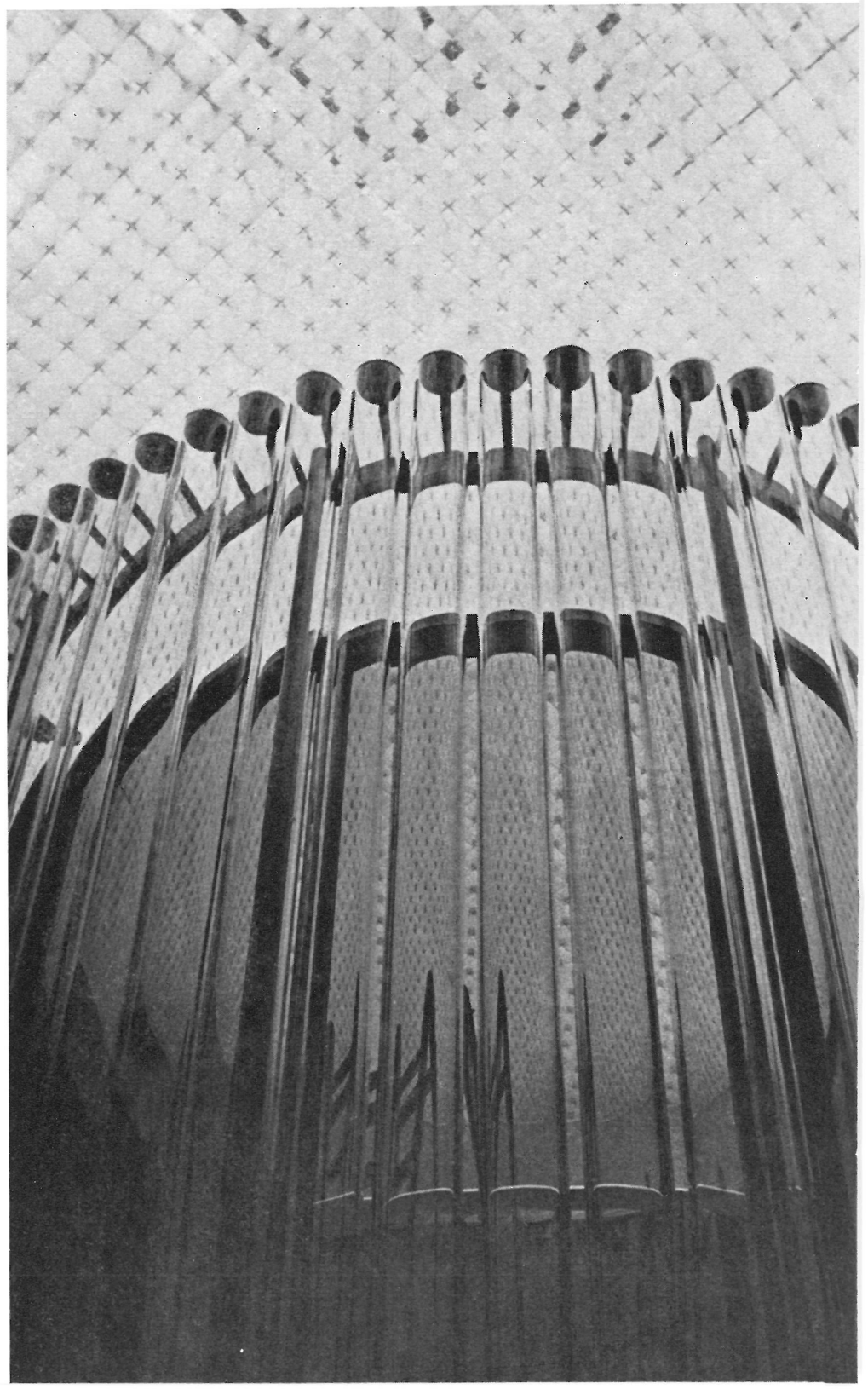


Así, ya en la fase de proyecto, estábamos decididos por un museo cerrado, partiendo de la premisa de que las salas de exposición ciegas proporcionan al visitante la calma y el aislamiento necesarios para concentrar su atención en las obras exhibidas. Además, la naturaleza en Hövikodden es muy bella, y hubiera podido desviar la vista del visitante, convirtiéndose en un factor concurrente peligroso para la adecuada visión de la exposición.

Esta es la razón del porqué, hacia el sur, diseñamos unas edificaciones «masivas y cerradas»; grandes salas de exposición irregulares y a las que intentamos imprimir un cierto dinamismo que invita al movimiento, y cuyo carácter y contenido cambia a medida que se avanza; este dinamismo pensamos lo hemos alcanzado, evitando todo sistema de composición basado en el ángulo recto.

Dos de las salas de pintura precisaban luz diurna para las exhibiciones de carácter permanente y, como a nuestro juicio, la luz lateral no era aconsejable, evitamos las ventanas, y el alumbrado con luz de día se consigue mediante la luz - cenital- que penetra a través de las cúpulas translúcidas existentes en la cubierta; el cielorraso construido debajo de la cubierta difunde la luz que recibe y adopta el aspecto de una superficie lisa y neutra, efecto logrado mediante el empleo de planchas de poliéster - que forman un cuadriculado- y difunden la luz de una manera especial.

Para componer el conjunto jugamos con una serie de bloques masivos, en los que contrastan la sensación de "pesadez» de los muros - hormigón- y la de «ligereza» de las cubiertas -revestidas de aluminio-.

La topografía y naturaleza del terreno, de una belleza excepcional, contribuyeron asimismo a nuestra creación. Hemos intentado el dar una respuesta arquitectónica a los rasgos característicos del mismo; así, las salas de exposición adoptan la forma de prismas sobre la parte accidentada, al sur, mientras que hemos ubicado el restaurante, los despachos, apartamentos, sala de conferencias, y biblioteca, etc., al noroeste, zona en la que la topografía del terreno es más tranquila y permitía un desarrollo horizontal de la edificación.

Los muros de fachadas y del interior del hall principal han sido construidos a base de hormigón vertido in situ, $\mathrm{y}$ a sus paramentos se les ha conferido un posterior «acabado»; sobre estos muros se apoya la estructura de la cubierta, constituida por una serie de vigas transversales, entre las que, en la zona de las salas de exposición, están situadas las cúpulas mencionadas anteriormente y debajo cuelga el cielorraso difusor; y en la zona del hall, restaurante, ala de administración, etc., descansa sobre las vigas de hormigón un forjado también de hormigón, del que cuelga el cielorraso constituido por paneles perforados de aluminio barnizados con laca blanca.

Dispone el conjunto de una amplia red de instalaciones especiales; así, todas las salas de exposición, restaurante y almacenes están climatizados.

El alumbrado artificial de las salas de exposición - cenital - ha sido conseguido utilizando fuentes luminosas de neón, adecuadamente estudiadas para conservar la armonía de los colores de las obras exhibidas; en las salas dedicadas a la celebración de exposiciones temporales se ha instalado un sistema de alumbrado especial específico para la exhibición de obras pictóricas. Igualmente, se ha instalado un sistema de iluminación especial, a base de proyectores regulables, en el teatro y sala de conciertos. Las salas con cielorraso de aluminio reciben iluminación diurna a través de las vidrieras, y la iluminación artificial se consigue mediante proyectores ocultos en los paneles del cielorraso.

Fue resuelto eficazmente el problema de la acústica en las salas de exposición; y con el fin de crear una atmósfera agradable en el hall, restaurante y oficinas, fueron adoptadas las oportunas medidas para obtener un adecuado grado de aislamiento acústico, a lo que ayuda las perforaciones que presentan los paneles de aluminio de los cielorrasos y la capa de material aislante extendida.

La sala de teatro y conciertos está especialmente preparada, con dispositivos especiales que regulan la absorción o amplificación del sonido.

Un sistema de alarma modernísimo, así como un sistema de control por televisión, forman parte de las instalaciones de seguridad.

Los paramentos interiores de las salas de exposición van tapizados con arpillera pintada de blanco; y el suelo está pavimentado con moqueta.

Unas consideraciones finales: Se asocia habitualmente a la palabra museo, un lugar en el que se conservan objetos de ayer y del pasado; pues bien, los fundadores de este museo han pretendido que éste, de Hövikodden, contribuya más bien a preservar el futuro; entendiendo que lo más importante es salvaguardar, cuando se trata de arte, la facultad de constante renovación, avivar la necesidad de buscar siempre el elemento positivo en la novedad, y de contemplar toda nueva obra con amplia apertura de espíritu, libre de prejuicios y convencionalismos. 
Estiman asimismo que la tradición es algo excelente e indispensable, pero que no debe forzarnos a sumergirnos y encerrarnos en ella y a mirar hacia atrás, sino que, por el contrario, la tradición debe de ser una fuerza que nos impulse hacia adelante, una especie de mantillo que haga brotar el elemento nuevo y ayude a revelarlo.

Gracias a estos puntos de vista, a esta apertura de espíritu, demostrada tanto en la selección de obras de arte a exhibir cómo en la creación de esta Institución, se abren perspectivas insospechadas en la vida cultural de Noruega. La colección de trofeos de Sonja Henie, expuesta en una sala especial, testimonio de su voluntad de triunfo, parece querer contagiar al ambiente de su continuado instinto de superación.

Traducido y adaptado por J. M. Rubio.

\section{mésumá O summmary}

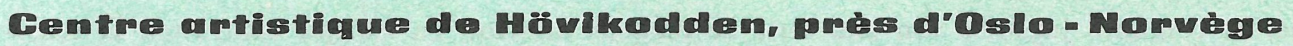

Eikvar et Engebretsen, architectes

Ce grand centre artistique, bâti à $12 \mathrm{~km}$ environ d'Oslo, est destiné non seulement à l'exposition de travaux plastiques, mais aussi à la recherche dans toutes les branches de l'art contemporain. Parmi ses installations figurent un musée, un théâtre, une salle de concerts et de conférences, une bibliothèque, une salle d'études, ainsi que des chambres pour les boursiers et les employés.

Pour composer cet ensemble, les architectes ont joué avec une série de blocs massifs, mettant en contraste la sensation de «lourdeur» suggérée par les murs et la sensation de «légèreté» des couvertures. Ils obtiennent ainsi une composition libre et dynamique, sans perdre toutefois le sens d'unité de l'ensemble.

Construit par la Fondation de Sonja Henie et Niels Onstad, cet organisme constitue un véritable forum, où la parenté entre les divers arts est rendue tangible, et un lieu largement ouvert aux échanges entre des tendances très variées.

\section{Artistic Comtre of Hövikoddem, near OSlo, Nomway}

Eikvar \& Engebretsen, architects

At about $12 \mathrm{kms}$ from Oslo this great artistic centre has been built, which is not only devoted to the exhibition of plastic works of art, but is also involved in the research of all aspects of contemporary art. Its installations include a museum, a theatre, lecture and concert halls, library, study rooms, as well as living quarters for scholarship students and the staff.

To achieve their purpose, the architects combined a variety of massive volumes, in which a contrast is achieved between the weightiness suggested by the walls, and the lightness of the roofs. The total design is uninhibited and dynamic, without weakening the sense of unity of the whole.

It has been built by the Sonja Henie and Niels Onstad foundation, and it constitutes a true forum for the various arts, and a meeting place for the exchange of influences between very diverse tendencies.

\section{Kumstzentrum in Hövikod den, Oslo/Nomwegen}

Eikvar und Engebretsen, Architekten

Circa $12 \mathrm{Km}$ von Oslo entfernt ist dieses grosse Kunstgebäude errichtet worden, welches nicht nur die Ausstellung von Plastiken bezweckt sondern auch die Erforschung der verschiedenen Kunstzweige in unserer Zeit. Dieser Bau zählt zu seinen Einrichtungen ein Museum, ein Theater, einen Konzert- und Konferenzsaal, eine Bibliothek, einen Lesesaal und Unterkünfte für Stipendiate und Angestellte.

Bei der Zusammenstellung des Gesamtbaues behandelten die Architekten eine Reihe massiver Blöcke; die von den Mauern ausgestrahlte "Schwere» kontrastiert mit der «Leichtigkeit» der Dächer, indem bei jeder Einzelheit die $\mathrm{Zu}$ sammengehörigkeit des Ganzen bewährt wird. Das Ergebnis ist eine freie und dynamische Komposition.

Im Auftrag der Stiftung Sonja Henie und Niels Onstad erbaut, ist dieses Haus ein wahrhaftiges Forum in dem die Verwandtschaft der verschiedenen Künste greifbar wird und Heim der offenen Tür für den Gedankenaustausch der unterschiedlichsten Gesinnungen. 